\title{
Real-Time Activity Recognition Technique for Surveillance Camera and Implementation on Digital Signal Processor
}

\author{
R. Karthikeyan \\ Assistant Professor \\ Department of Electrical and \\ Electronics Engineering \\ Jaya Engineering College
}

\author{
P.Mahalakshmi \\ Assistant Professor \\ Department of Electronics and \\ Communication Engineering \\ Sakthi engineering college
}

\author{
N.Gowri Shankar \\ Assistant Professor \\ Department of Electronics and \\ Communication Engineering \\ Meenakshi Sundararajan \\ Engineering College
}

\begin{abstract}
Intelligent Video Surveillance allows users to easily monitor the secure areas with surveillance cameras, and thus eliminating the need for manual work and saves the huge monitoring costs. A novel method for object tracking, based on image segmentation is proposed to automatically recognize activities around restricted area to improve safety and security of the servicing area by multiplexing hundreds of video streams in real time. Key component for the proposed system includes background learning and updating, foreground segmentation, features extraction, and decision-making process. The proposed method uses adaptive background subtraction techniques to handle illumination changes to improve the performance of the video surveillance and videoenable operations. The algorithms are simulated using MATLAB tool to verify its stability in various conditions by giving various input video samples and its output is taken as benchmark for real time implementation in DSP processor.
\end{abstract}

\section{Keywords}

Video Analytics, image segmentation, tracking, video surveillance, Background Subtraction

\section{INTRODUCTION}

Manual analysis of video is labor-intensive, fatiguing, and prone to errors [1]. Additionally, research indicates that there are severe limitations in the ability of humans to monitor simultaneous signals [2].The ability to quickly search large volumes of existing video or monitor real-time footage will provide dramatic capabilities to transit agencies. Some drawbacks of video analytic systems are their vulnerability to environmental variables, such as detrimental lighting conditions and weather. These adverse conditions can trigger false alarms, which may become a source of frustration for the user. Conversely, a human analyst may use judgment and training to determine if an alarm should be raised for a wider range of scenarios. Video analytic algorithms often are sensitive to parameters and initial calibration. Event detection performance typically depends on this calibration process. It is difficult to achieve a good balance between event detection and false alarms [3].

The entire paper is organized as follows: Section 2 introduces all the procedure about background subtraction and temporal differencing method. Section 3 described an improved proposed method. Section 4 presents the implementing model and Section 5 presents experimental results of the Real-Time Activity Recognition Technique .Section V concludes the research work.

\section{BACKGROUND SUBTRACTION AND TEMPORAL DIFFERENCING}

A popular object segmentation strategy is background subtraction. Background subtraction compares an image with an estimate of the image as if it contained no objects of interest [4]. It extracts foreground objects from regions where there is significant difference between the observed and the estimated image. Common algorithms include methods by Heikkila and Olli [5], Stauffer and Grimson (Adaptive Gaussian Mixture Model or GMM) [6], Halevy [7], and Cutler [8]. A detailed general survey of image change algorithms can be found in Radke et al [9]. GMM is one of the most commonly-used methods for background subtraction in visual surveillance applications for fixed cameras. A mixture of Gaussians is maintained for each pixel in the image. As time passes, new pixel values update the mixture of Gaussians using an online K-means approach. The estimation update is used to account for illumination changes, slight sensor movement, and noise [10]. Nevertheless, transit surveillance researchers continue to emphasize the importance of robust background subtraction methods [11] and online construction and adaptive background models. A large number of recent background subtraction methods improve on prior existing methods by modeling the statistical behavior of a particular domain or by using a combination of methods.

\section{PROPOSED METHOD}

The algorithms are first simulated using MATLAB tool to verify its stability in various conditions by giving various input video samples and its output is taken as benchmark for real time implementation. The real time implementation of the algorithms is done by using the DSP processor. Figure 1 shows the simulation flow of the proposed system where the input for the method is given to the MATLAB tool and output is viewed in the system.

Figure 2 shows the simulation flow diagram of the real time implementation using DM355 Texas instrument development board. The real-time input is given to the board by using analog surveillance camera. To verify the stability of the system the board is connected to the PC and the algorithm is verified by using various input samples downloaded from the internet under various illumination conditions. 
Simulation flow:
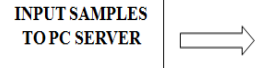
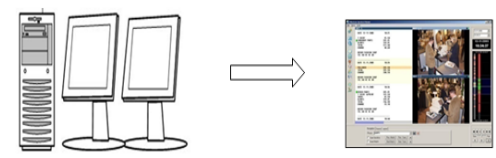

OUTPUT

SNAPSHOT OF MATLAB TOOI
PC SERVER RUNNING SOFTWARE

5. The Object Representation is done by rectangular shape to cover the object boundary to represent the object.

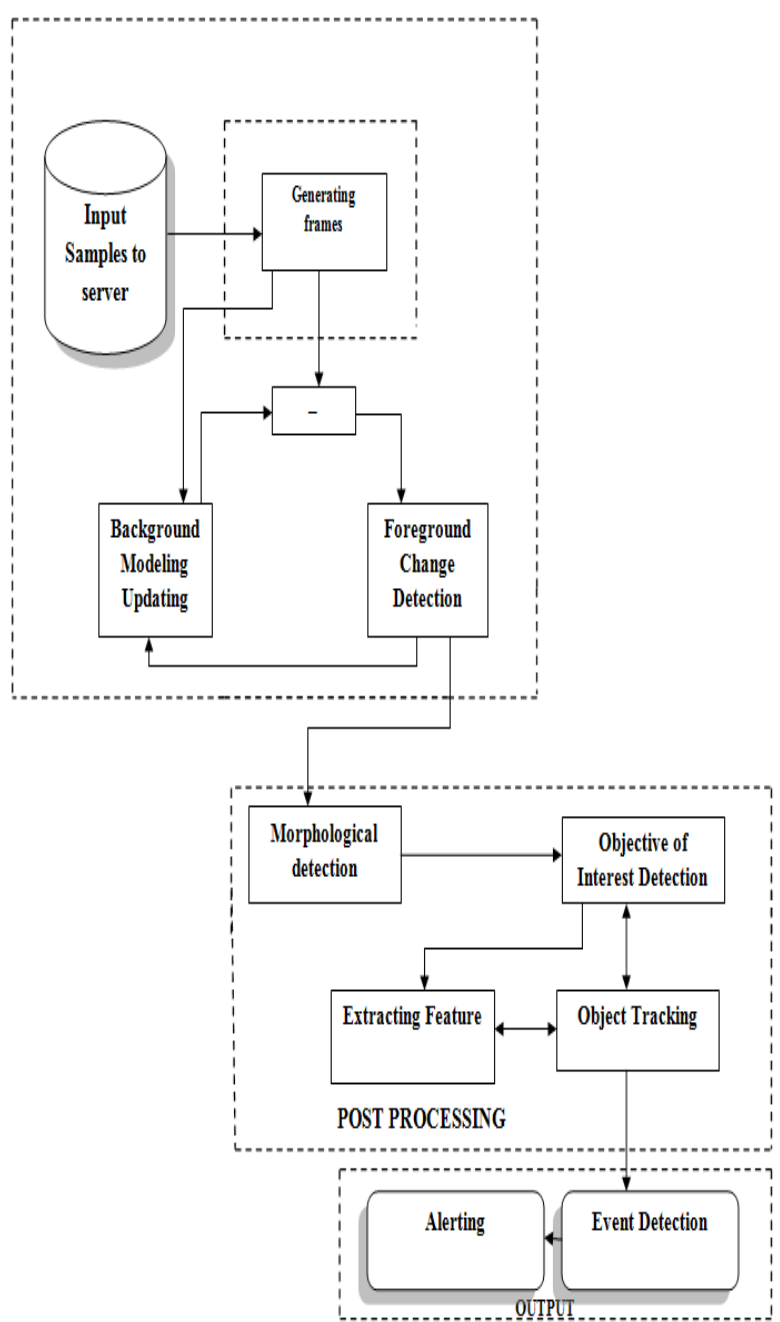

Figure4: Real time implementing flow diagram

The real time implementing method uses DM355 board of Texas instruments which contains TMS320C6x Processor for processing the videos. An analog surveillance camera is connected with the board and the algorithm is verified. To verify the algorithm stability the board is connected to the system and video are collected from the databases under

Figure 3: Implementing System Model.

\subsection{Algorithm flow}

The flow of algorithm is shown in Fig.4 as a flow chart and the process is explained in steps,

1. The video input received from the Analog Camera is converted into frames.

2. Object detection is done by using frame difference method.

3. Post processing is done to enhance the segmented image in order to improve the required result.

4. Feature Selection is based on Centroid of an object to plot the location of non-rigid body (objects) with frame to frame.

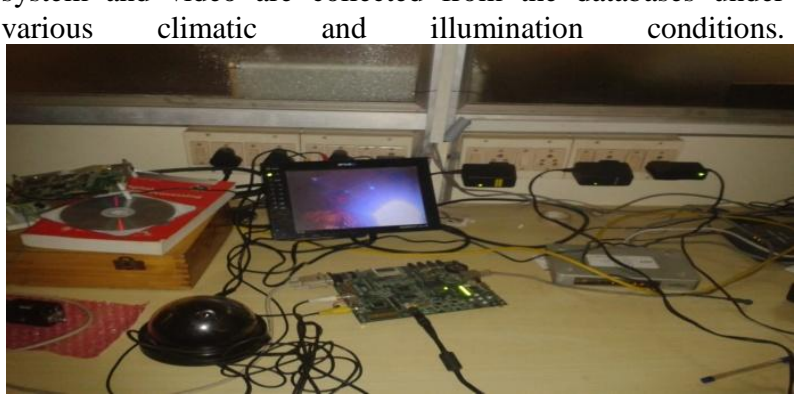

Figure 5: Snapshot of the Real time implementing method in the Lab.

Code Composer Studio ${ }^{\mathrm{TM}}$ (CCStudio) an integrated development environment (IDE) for Texas Instruments' (TI) embedded processor families is used to verify the code which 
is fused in to the processor. To boot the processor RJ45 cable is used to connect the board and system through a hub. RS232 cable is connected between the system and board to transfer the video samples. A 10 inch TFT Monitor is connected to the development board to view the processed video output.

\section{RESULTS}

In this chapter all real time results for all the techniques are explained. Initially the moving objects in video images are tracked based on image segmentation, background subtraction and object detection techniques. Algorithm for Unattended baggage and Motion detection and Intrusion Detection is discussed in detail. First the real time video input is given to the system and results are analyzed. To verify the reliability of the system the video samples downloaded from the various databases are given to the system through PC and frames of the output videos displayed.

\subsection{Unattended baggage and Motion detection}

The input video sequence is shown as frames in Fig 6. The results obtained are shown in Fig 7 and the motion detection is identified and marked in yellow lines and the unattended baggage found is marked in red color.

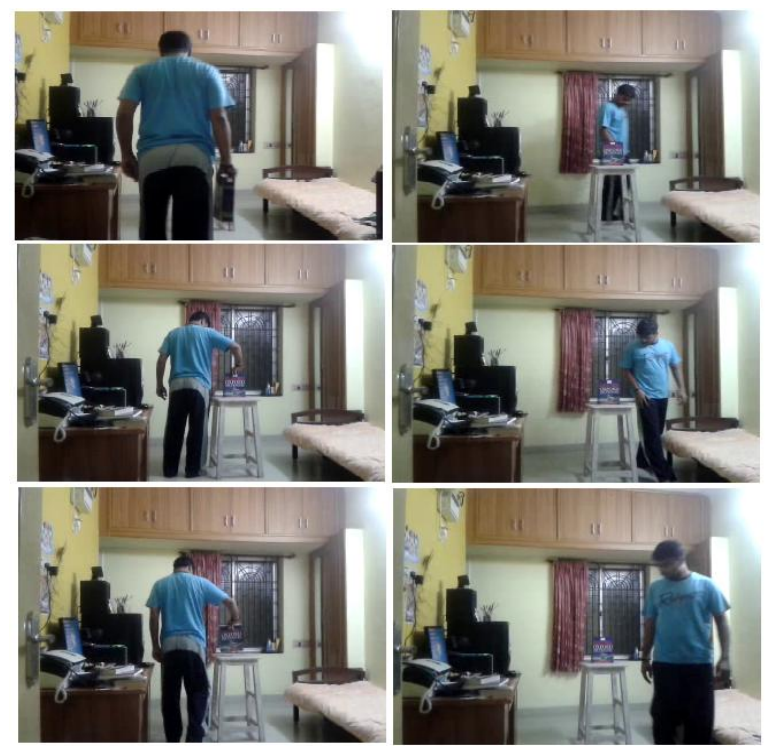

Figure 6: The input video sequence is shown as frames.
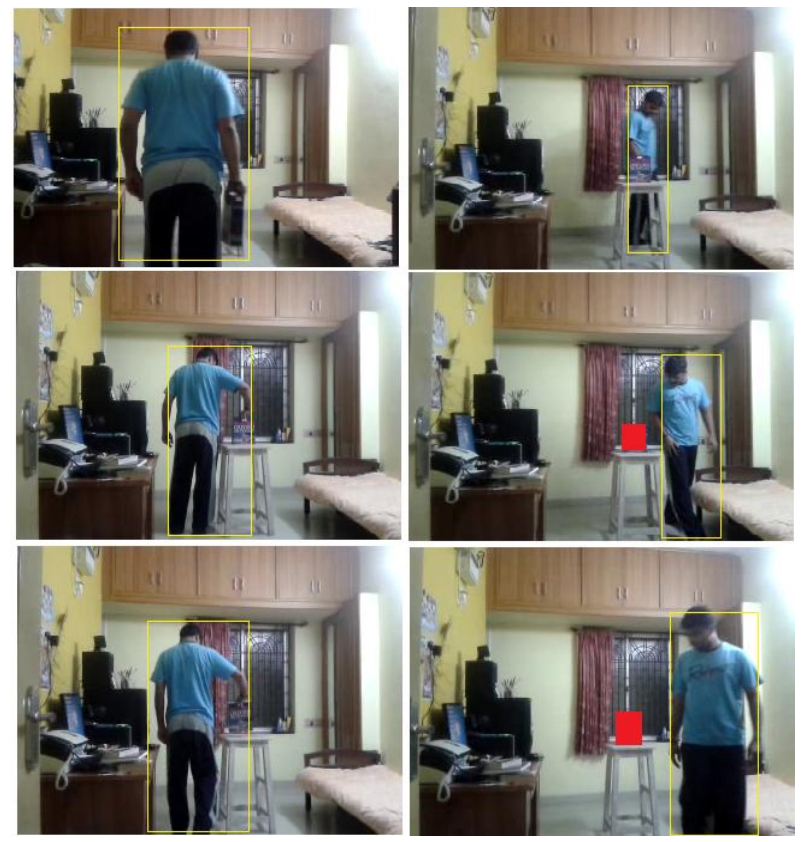

Figure 7: The Output video sequence for Motion detection and unattended baggage detection.

\subsection{Intrusion Detection}

The results obtained are shown in Fig 8 and the restricted area is marked in red color, when intruder passes in the restricted area the color changes to green.
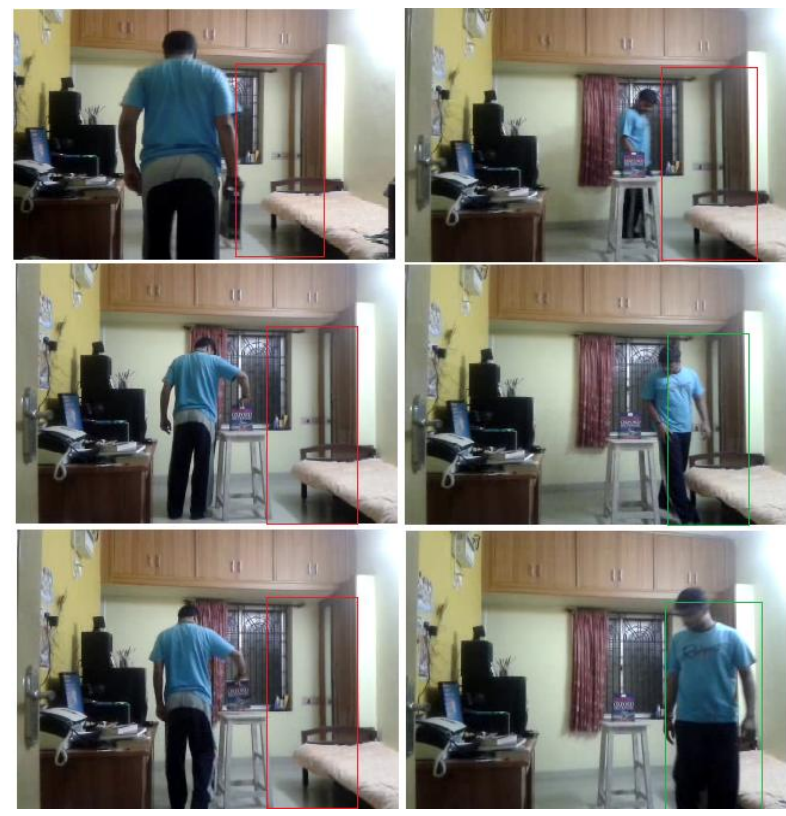

Figure 8: The Output video sequence for Intrusion detection. 


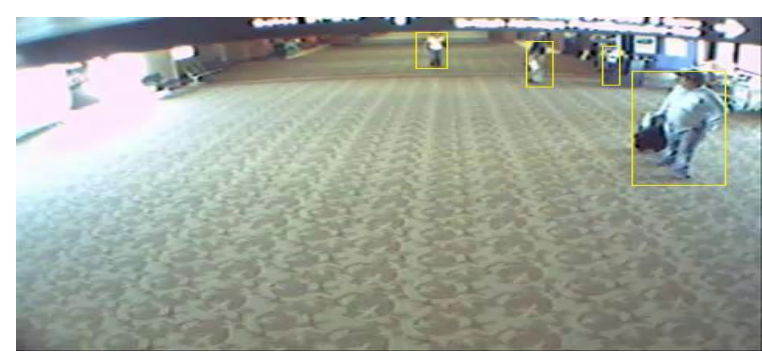

Figure 9: The Output video sequence for Motion detection

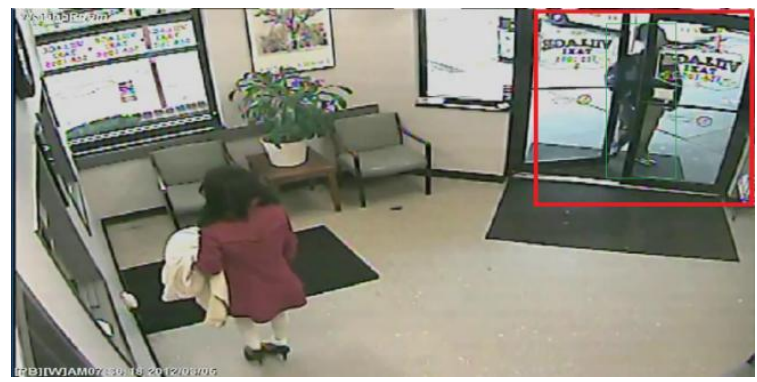

Figure 10: The Output video sequence for Intrusion detection.

\section{CONCLUSION}

An object tracking algorithm for video, based on image segmentation and pattern matching of the segmented objects between frames in a simple feature space is proposed. Simulation and real time results for frame sequences with video sequences verify the suitability of the algorithm for reliable moving object tracking. The proposed method uses single camera method it can be further extended to multiple camera method to enhance the security in surveillance system. The proposed algorithm can be optimized further to reduce the processing time and can be implemented using other processors to verify its processing speed.

\section{REFERENCES}

[1] M. Valera and S.A. Velastin, "Intelligent distributed surveillance systems: a review." IEEE Proc. Vision, Image and Signal Processing, vol. 2, pp. 192-204, 2005.

[2] N. Sulman, T. Sanocki, D. Goldgof, and R. Kasturi, "How effective is human video surveillance performance?," Int. Conference Pattern Recognition, pp. $1-3,2008$

[3] W. Hu, T. Tan, L. Wang, and S. Maybank "A survey on visual surveillance of object motion and behaviors," IEEE Trans. Systems, Man, and Cybernetics Part C, vol. 34, no. 3, pp. 334-352, 2004.

[4] Massimo Piccardi "Background subtraction techniques: a review", Computer Vision Research Group (CVRG), University of Technology, Sydney (UTS), The ARC Centre of Excellence for Autonomous Systems (CAS) Faculty of Engineering, UTS, April 15,2004

[5] J. Heikkila and O. Silven, "A real-time system for monitoring of cyclists and pedestrians," IEEE Workshop on Visual Surveillance, pp. 74-81, 1999.

[6] C. Stauffer and W. E. L. Grimson, "Adaptive background mixture models for real-time tracking," IEEE Int. Conference on Computer Vision and Pattern Recognition, vol. 2, pp. 246-252, 1999.

[7] G. Halevy and D.Weinshall, "Motion of disturbances: detection and tracking of multibody non-rigid motion," IEEE Int. Conference on Computer Vision and Patter Recognition, pp. 897-902, 1997.

[8] R. Cutler and L. Davis, "View-based detection and analysis of periodic motion," Int. Conference on Pattern Recognition, pp. 495-500, 1998

[9] R.J. Radke, S. Andra, O. Al-Kofahi, and B. Roysam, "Image change detection algorithms: a systematic survey," IEEE Trans. on Image Processing, vol. 14, no. 3, pp. 294-307, 2005

[10] A. Dominguez-Caneda, C. Urdiales, and F. Sandoval, "Dynamic background subtraction for object extraction using virtual reality based prediction," Electrotechnical Conference (MELECON), pp. 466-469, 2006.

[11] S-C. Cheung and C. Kamath, "Robust background subtraction with foreground validation for Urban Traffic Video," EURASIP Journal on Applied Signal Processing, vol. 14, pp. 1-11, 2005.

[12] Rafael C Gonzalez, Richard E Woods, "Digital Image Processing". 